\title{
CARACTERIZAÇÃO NUTRICIONAL E RESPOSTA SENSORIAL DE PÃES DE MEL COM ALTO TEOR DE FIBRA ALIMENTAR ELABORADOS COM FARINHAS DE SUBPRODUTOS DO PROCESSAMENTO DE FRUTAS
}

\author{
FERNANDA TEIXEIRA MACAGNAN * \\ FERNANDA ALINE DE MOURA ** \\ LUCCIELLI RODRIGUES DOS SANTOS *** \\ MARÍLIA BIZZANI *** \\ LEILA PICOLLI DA SILVA ****
}

\begin{abstract}
O objetivo deste trabalho foi avaliar a qualidade nutricional e sensorial de pães de mel enriquecidos com farinhas de bagaço de maçã, de bagaço de laranja e de casca de maracujá. Com o intuito de viabilizar o uso desses ingredientes alternativos e de baixo custo como fontes de fibra alimentar testou-se a substituição de $15 \%$ da farinha de trigo da formulação padrão pelas farinhas dos respectivos subprodutos de frutas. As formulações teste apresentaram alto teor de fibra alimentar, especialmente da fração solúvel, maior umidade e reduzido valor calórico. A formulação adicionada de bagaço de maçã destacou-se entre as demais pelo aporte significativamente superior de fibra $(9,61 \%)$. Os resultados obtidos na análise sensorial dos pães indicaram boa aceitabilidade desses produtos para todos os parâmetros sensoriais avaliados (cor, aroma, sabor e textura), perfazendo índice médio de aceitação global entre 77,4 \% e $80,3 \%$. Além disso, verificou-se intenção de compra positiva, com $39,3 \%$ a $44,6 \%$ das respostas concentradas no termo hedônico "possivelmente compraria", sem diferença significativa entre as formulações teste. Os resultados indicaram a possibilidade de uso desses subprodutos de frutas como ingredientes fibrosos para serem usados na formulação de produtos alimentícios diferenciados, como o pão de mel, por melhorarem significativamente o aporte de fibras (principalmente em relação à fração solúvel), o que contribuirá para o combate do desperdício de nutrientes potenciais e para a promoção da saúde do consumidor.
\end{abstract}

PALAVRAS-CHAVE: BAGAÇO DE MAÇÃ; CASCA DE MARACUJÁ; BAGAÇO DE LARANJA; FIBRA SOLÚVEL; ALIMENTO FUNCIONAL; ANÁLISE SENSORIAL.

* Farmacêutica, Mestranda em Ciência e Tecnologia dos Alimentos, Departamento de Tecnologia e Ciência dos Alimentos (DTCA), Universidade Federal de Santa Maria (UFSM), Santa Maria, RS, Brasil (e-mail: femacagnan@yahoo.com.br).

** Nutricionista, Doutoranda em Ciência e Tecnologia dos Alimentos, DTCA, UFSM, Santa Maria, RS, Brasil (e-mail: fernandamoura_nut@yahoo.com.br).

*** Graduandas em Tecnologia dos Alimentos, DTCA, UFSM, Santa Maria, RS, Brasil (e-mail: lutyrodrigues@ yahoo.com.br; maribizzani@hotmail.com).

**** Engenheira Agrônoma, Professora Adjunto, Departamento de Zootecnia, UFSM, Santa Maria, RS, Brasil (e-mail: leilasliva@yahoo.com.br). 


\section{INTRODUÇÃO}

Os efeitos fisiológicos e metabólicos decorrentes do consumo adequado de fibra alimentar na prevenção de inúmeras doenças crônico-degenerativas estão bem consolidados pela comunidade científica (FIETZ e SALGADO, 1999; MATTOS e MARTINS, 2000; GONÇALVES et al., 2007). Contudo, sua ingestão pela população brasileira mostrou-se inferior às recomendações nutricionais, provavelmente devido à substituição de alimentos naturais, fontes de fibras, por alimentos industrializados e pobres nutricionalmente (MENEZES, GIUNTINI e LAJOLO, 2001).

Várias campanhas de saúde preventiva têm demostrado a necessidade de suprir o consumo insuficiente de fibra alimentar, motivando a indústria a desenvolver alimentos enriquecidos com fibra, agregando valor nutricional aos seus produtos, além de contribuir para a promoção da saúde da população (CERQUEIRA et al., 2008). Assim, torna-se importante que novos ingredientes e formulações sejam estudados a fim de ampliar esse segmento alimentício, atendendo sempre aos atributos sensoriais exigidos pelo consumidor.

O beneficiamento de frutas para a produção de sucos gera quantidade apreciável de subprodutos, tais como casca de maracujá, bagaço de laranja e bagaço de maçã (BORTOLUZZI, 2009; CANTERI, 2010, RAUPP et al., 2000). A utilização desses materiais secundários do processamento de frutas como ingredientes na formulação de alimentos, além de diminuir o desperdício e evitar a contaminação ambiental pelo descarte incorreto desses materiais, poderá facilitar o acesso da população de baixa renda a alimentos funcionais. Esses potenciais ingredientes alimentícios de baixo custo ainda se destacam por apresentarem quantidade apreciável de pectina, polissacarídeo solúvel que está intimamente associado à maioria dos efeitos benéficos da fibra ao organismo humano (FIETZ e SALGADO, 1999; RIQUE, SOARES e MEIRELLES, 2002; CANTERI et al., 2012).

As fibras oriundas de frutas apresentam vantagens tecnológicas em relação às fibras de cereais, amplamente utilizadas para enriquecer os alimentos. Tais vantagens ocorrem devido ao maior teor de fibra alimentar e sua fração solúvel, à maior capacidade de retenção de água e gordura, ao menor conteúdo de ácido fítico e menor valor calórico (LARRAURI, 1999). Admite-se assim, tanto sob o ponto de vista nutricional quanto tecnológico, a importante aplicabilidade desses subprodutos na indústria de alimentos, como alternativa para as fibras convencionais.

O presente trabalho foi conduzido com o objetivo de avaliar a composição química, a aceitabilidade e a intenção de compra de pães de mel adicionados de farinhas de bagaço de maçã, bagaço de laranja e de casca de maracujá em substituição parcial à farinha de trigo.

\section{MATERIAL E MÉTODOS}

\subsection{MATÉRIAS-PRIMAS}

As amostras de bagaço de maçã (mistura das variedades Gala e Fuji) e bagaço de laranja (mistura das variedades Rubi e Hamlin), obtidas após a etapa de prensagem para fabricação do suco, foram fornecidas pela Indústria Fischer Sucos (Videira/SC, Brasil). No laboratório, foram descongeladas, higienizadas por imersão em solução clorada (200 ppm/15 min) e enxaguadas com água corrente. As cascas de maracujá azedo foram separadas manualmente dos frutos, adquiridos no comércio de Santa Maria (RS/ Brasil) e higienizados em solução clorada antes da retirada da casca.

Os subprodutos de frutas foram pré-secos em estufa de circulação de $\operatorname{ar}\left(55^{\circ} \mathrm{C} / 48\right.$ horas), moídos em micromoinho (marca Marconi®, $27.000 \mathrm{rpm}$, partículas $<1 \mathrm{~mm}$ ), e as farinhas resultantes, armazenadas em sacos plásticos à temperatura de $-18{ }^{\circ} \mathrm{C}$ até o momento da elaboração dos pães de mel. A composição química das farinhas dos subprodutos de frutas utilizadas está descrita na Tabela 1. Os demais ingredientes utilizados nas formulações foram obtidos no comércio da cidade de Santa Maria. 
TABELA 1 - COMPOSIÇÃO QUÍMICA DAS FARINHAS DE BAGAÇO DE MAÇÃ (BM), BAGAÇO

DE LARANJA (BL) E CASCA DE MARACUJÁ (CM) UTILIZADAS NAS FORMULAÇÕES

DOS PÃES DE MEL, EXPRESSAS EM MATÉRIA SECA (MS)

\begin{tabular}{lccc}
\hline \multicolumn{1}{c}{ DETERMINAÇÕES } & BM & BL & CM \\
\hline Umidade (\%) & 5,64 & 8,45 & 7,38 \\
& & $\%$ MS & \\
Matéria Mineral & 1,26 & 2,97 & 6,04 \\
Proteína & 6,98 & 4,89 & 5,26 \\
Lipídios & 8,19 & 4,21 & 1,26 \\
Fibra alimentar total & 76,84 & 54,82 & 62,65 \\
Fibra alimentar insolúvel & 57,87 & 29,65 & 43,43 \\
Fibra alimentar solúvel & 18,97 & 25,17 & 19,22 \\
Carboidratos não fibrosos & 6,72 & 33,12 & 24,88 \\
Pectina (pectato de cálcio) & 17,31 & 12,38 & 15,58 \\
\hline
\end{tabular}

\subsection{ELABORAÇÃO DOS PÃES DE MEL}

Foram elaboradas três formulações teste de pães de mel, tendo como base para análise de composição química a formulação padrão (FP) sem adição dos subprodutos. Incorporou-se a cada formulação teste $15 \%$ de farinha do subproduto de fruta correspondente em substituição à farinha de trigo da FP (sem alteração na quantidade dos demais ingredientes), obtendo-se as formulações FBM (adicionada de bagaço de maçã), FBL (adicionada de bagaço de laranja) e FCM (adicionada de casca de maracujá).

Para compor as formulações teste, além das farinhas dos subprodutos de frutas e de trigo, foram usados chocolate em pó, fermento químico, açúcar mascavo, açúcar invertido, mel, margarina, chocolate meio amargo e chocolate branco, bicarbonato, canela, ovo e água, conforme as proporções representadas na Tabela 2.

Os ingredientes foram pesados separadamente em frascos plásticos, previamente identificados. Inicialmente, foram adicionados $20 \mathrm{~mL}$ de água a cada uma das farinhas dos subprodutos e após homogeneização manteve-se a mistura em repouso por 30 minutos para melhor hidratação da fibra. Aqueceu-se o açúcar mascavo com o restante da água por 1 minuto. Depois de resfriar, incorporou-se à calda formada, o mel, a canela, o chocolate em pó, a(s) farinha(s), a margarina, o ovo, o bicarbonato e o fermento químico, misturando-se devidamente cada ingrediente a fim de obter massa homogênea. Após descanso por 15 minutos, a massa foi estendida com auxílio de rolo de alumínio, moldando-se os pães de mel em formato circular com aproximadamente $1,0 \mathrm{~cm}$ de altura e $3,2 \mathrm{~cm}$ de diâmetro. Os pães foram assados em forno elétrico pré-aquecido a $200^{\circ} \mathrm{C}$ por 20 minutos, resfriados até a temperatura ambiente e decorados com chocolate.

\subsection{ANÁLISE DA COMPOSIÇÃO QUÍMICA DOS PÃES DE MEL}

Para caracterização química das amostras de pães de mel foram realizadas, em triplicata, as seguintes análises: umidade em estufa a $105{ }^{\circ} \mathrm{C}$ por 12 horas; matéria mineral por incineração em mufla a $550{ }^{\circ} \mathrm{C}$ por 5 horas; proteína bruta por meio da determinação de nitrogênio pelo método 
de Kjeldahl $(\mathrm{N} \times 6,25)$, conforme as técnicas descritas pela AOAC (1995). O conteúdo de lipídios foi obtido pelo método de Bligh e Dyer (1959) e o teor de fibra alimentar determinado de acordo com o método enzimático 991.43 da AOAC (1995), incluindo fibra alimentar total e sua fração insolúvel, assim como a fibra solúvel (diferença entre as duas anteriores).

Estimou-se a fração de carboidratos não fibrosos por diferença, subtraindo-se de cem os valores obtidos para umidade, proteína, lipídio, fibra alimentar total e matéria mineral. Calculou-se o valor calórico total das formulações usando os valores de conversão para carboidratos $\left(4,0 \mathrm{kcal} . \mathrm{g}^{-1}\right)$, lipídios $\left(9,0 \mathrm{kcal} . \mathrm{g}^{-1}\right)$ e proteína $\left(4,0 \mathrm{kcal} . \mathrm{g}^{-1}\right)$ (BRASIL, 2003).

TABELA 2 - FORMULAÇÕES ADOTADAS PARA OS PÃES DE MEL

\begin{tabular}{lcccc}
\hline \multicolumn{1}{c}{ Ingredientes \% } & FP* & FBM & FBL* & FCM $^{*}$ \\
\hline Farinha de Trigo & 100 & 85 & 85 & 85 \\
Farinha de Bagaço de Maçã & - & 15 & - & - \\
Farinha de Bagaço de Laranja & - & - & 15 & - \\
Farinha de Casca de Maracujá & - & - & - & 15 \\
\hline Chocolate em pó & 2,50 & 2,50 & 2,50 & 2,50 \\
Fermento Químico & 2,50 & 2,50 & 2,50 & 2,50 \\
Bicarbonato & 1,00 & 1,00 & 1,00 & 1,00 \\
Açúcar Mascavo & 30,00 & 30,00 & 30,00 & 30,00 \\
Açúcar Invertido & 10,00 & 10,00 & 10,00 & 10,00 \\
Mel & 20,00 & 20,00 & 20,00 & 20,00 \\
Margarina & 4,00 & 4,00 & 4,00 & 4,00 \\
Ovo & 4,50 & 4,50 & 4,50 & 4,50 \\
Canela & 0,25 & 0,25 & 0,25 & 0,25 \\
Chocolate cobertura & 12,00 & 12,00 & 12,00 & 12,00 \\
Água & 30,00 & 30,00 & 30,00 & 30,00 \\
\hline
\end{tabular}

Nota: porcentagem dos ingredientes calculada em relação ao peso total das farinhas. * FP = formulação padrão, FBM = formulação adicionada de bagaço de maçã; FBL = formulação adicionada de bagaço de laranja; FCM = formulação adicionada de casca de maracujá.

\subsection{ANÁLISE SENSORIAL DOS PÃES DE MEL}

O presente estudo foi aprovado pelo Comitê de Ética em Pesquisa da Universidade Federal de Santa Maria (UFSM) em seus aspectos éticos e metodológicos, sob o protocolo de $n^{\circ}$ 03540112.5.0000.5346.

As formulações de pães de mel contendo as farinhas dos subprodutos de frutas foram submetidas ao teste de aceitabilidade e de intenção de compra. Conduziu-se a avaliação sensorial em cabines individuais, com 56 julgadores não treinados de ambos os sexos e faixa etária entre 18 e 49 anos.

As amostras, com aproximadamente $10 \mathrm{~g}$, foram oferecidas de forma aleatória e monádica, envoltas em filme plástico transparente de policloreto de vinila e codificadas com números de três dígitos correspondentes ao código de cada formulação.

Para o teste de aceitabilidade utilizou-se escala hedônica estruturada de sete pontos 
( 1 = desgostei muitíssimo, 4 = indiferente e 7 = gostei muitíssimo). 0 grupo de julgadores avaliou cada amostra, separadamente, quanto aos atributos cor, aroma, sabor e textura (sensação na boca), além da aceitação global dos produtos desenvolvidos.

O teste de intenção de compra foi realizado utilizando-se escala de cinco pontos, com nota máxima 5 correspondendo a "certamente compraria", nota mínima 1 referindo-se a "certamente não compraria" e nota 3 expressando "talvez comprasse/talvez não comprasse" (INSTITUTO ADOLFO LUTZ, 2008).

\subsection{ANÁLISE ESTATÍSTICA}

Os dados da caracterização química e do teste sensorial de aceitação dos pães de mel foram avaliados estatisticamente pela análise de variância e a comparação das médias das amostras pelo teste de Tukey ao nível de significância de $5 \%$, utilizando-se o programa Statistical Package for Social Science, versão 8.0 (SPSS, 1997). Os resultados da intenção de compra foram analisados por teste de frequência.

\section{RESULTADOS E DISCUSSÃO}

\subsection{COMPOSIÇÃO QUÍMICA}

A inclusão das farinhas de subprodutos de frutas aumentou significativamente os teores de fibra alimentar e de umidade das formulações teste em comparação à formulação padrão (Tabela 3). A presença de maior teor de fibra nos pães de mel auxiliou a retenção de água no produto após o cozimento, que pode estar relacionada à capacidade de hidratação da fibra, propriedade que influencia profundamente a textura final do produto (BORTOLUZZI, 2009; ELLEUCH et al., 2011). Resultados obtidos em outros estudos também verificaram elevada capacidade hidrofílica da fibra alimentar, a qual se mostrou diretamente relacionada ao seu teor no alimento (SILVA, BORGES e MARTINS, 2001; PEREZ e GERMANI, 2007; MAURO, SILVA e FREITAS, 2010).

A substituição de $15 \%$ da farinha de trigo pela farinha proveniente dos subprodutos de frutas aumentou, em média, 4,3 vezes o aporte de fibra alimentar nos pães de mel. Deve-se destacar que a formulação com bagaço de maçã apresentou 9,61 \% de fibra, 5,2 vezes mais que a FP. A FBM mostrou maior teor desse nutriente quando comparada a outras formulações de pães de mel enriquecidas com fontes de fibra, proveniente de grãos em substituição a $20 \%$ de farinha de trigo (POSSAMAI, 2005). Além disso, rótulos nutricionais de pães de mel comerciais mencionam teor de fibra alimentar entre 0 a 1 \% (POSSAMAI, 2005), fazendo com que as formulações propostas no presente estudo atendam às expectativas de consumidores que apreciam o produto e que primam pela qualidade nutricional de suas refeições.

Segundo a Portaria 27, da Secretaria de Vigilância Sanitária do Ministério da Saúde (BRASIL, 1998), os pães de mel elaborados com as farinas dos subprodutos de frutas poderiam ser classificados como produtos com alto teor de fibra, que devem conter no mínimo $6 \%$ desse nutriente para serem declarados como tal.

Levando em consideração as recomendações nutricionais propostas para a população brasileira de no mínimo $20 \mathrm{~g} /$ dia de fibra alimentar para jovens e adultos (MENEZES, GIUNTINI e LAJOLO, 2001), as formulações testadas poderiam contribuir consideravelmente para se atingir tal recomendação. A porção de $30 \mathrm{~g}$ (quantidade normalmente encontrada para pães de mel comerciais), correspondente a três unidades dos produtos desenvolvidos neste estudo, representa entre 9,9\% (FBL) e 14,4 \% (FBM) da ingestão diária recomendada para esse nutriente. A ingestão habitual de fibra alimentar torna-se relevante, devido aos seus efeitos fisiológicos benéficos na prevenção de inúmeras doenças degenerativas, como também na manutenção da regularidade das funções digestivas (LÓPEZ et al.,1997; MENEZES, GIUNTINI e LAJOLO, 2001; GONÇALVES et al., 2007)

A utilização das farinhas dos subprodutos nas formulações testadas também contribuiu significativamente para o aumento da fração solúvel da fibra, cujas propriedades físico-químicas 
(como o aumento da viscosidade do conteúdo intestinal e elevado grau de fermentabilidade) estão intimamente relacionadas com a maioria dos efeitos benéficos da fibra alimentar no organismo humano (LÓPEZ et al.,1997; FIETZ e SALGADO, 1999; RIQUE, SOARES e MEIRELLES, 2002). Entre esses efeitos estão o controle da glicemia pós-prandial e a diminuição do colesterol sérico, bem como o aumento do balanço microbiótico e a produção de ácidos graxos de cadeia curta, resultantes da fermentação da fibra e fundamentais para a manutenção da saúde intestinal (LÓPEZ et al.,1997; RIQUE, SOARES e MEIRELLES, 2002). Deve-se enfatizar que a contribuição da fibra solúvel no produto alimentício não pode ser observada com tanta intensidade quando se utilizam as fibras convencionais provenientes de cereais, pois essas são compostas (quase que na sua totalidade) pela fração insolúvel (LARRAURI, 1999).

A FBL e principalmente a FCM tiveram aumentados seus teores de minerais em comparação com as demais formulações $(p<0,05)$. Isso pode ser explicado pelo maior conteúdo desses nutrientes nas farinhas do bagaço de laranja e da casca de maracujá $(2,97 \%$ e 6,04 \% em base seca, respectivamente) em comparação às farinhas de bagaço de maçã e de trigo $(0,8 \%)$, conforme o NEPA (2011). Os resultados encontrados no presente trabalho para matéria mineral nas formulações de pães de mel $(1,54 \%$ a $1,82 \%)$ são próximos aos obtidos para pães de mel enriquecidos com farelo de trigo e linhaça (1,55 e 1,57 \% em base úmida, respectivamente) descritos no estudo de Possamai (2005).

O conteúdo proteico foi significativamente inferior para as formulações acrescidas das farinhas dos subprodutos de frutas. Esse resultado reflete o menor teor desse componente dietético nessas farinhas em comparação à farinha de trigo $\left(9,8 \mathrm{~g} 100 \mathrm{~g}^{-1}\right)$ (NEPA, 2011), que está presente em maior quantidade na formulação padrão. Assim, tais farinhas não poderiam ser incorporadas em formulações alimentícias com a finalidade única de melhorar o aporte proteico.

TABELA 3 - COMPOSIÇÃO QUÍMICA DAS FORMULAÇÕES DE PÃES DE MEL (\% BASE ÚMIDA)

\begin{tabular}{|c|c|c|c|c|}
\hline \multirow[t]{2}{*}{ DETERMINAÇÕES } & \multicolumn{4}{|c|}{ Formulações } \\
\hline & FP & FBM & FBL & FCM \\
\hline Umidade & $15,94 \pm 0,16^{c}$ & $22,61 \pm 0,07$ ab & $22,17 \pm 0,13^{b}$ & $22,93 \pm 0,21^{\mathrm{a}}$ \\
\hline Proteína & $5,83 \pm 0,11^{\mathrm{a}}$ & $5,10 \pm 0,01^{\mathrm{b}}$ & $4,74 \pm 0,02^{c}$ & $4,96 \pm 0,08^{b}$ \\
\hline Matéria Mineral & $1,50 \pm 0,06^{c}$ & $1,54 \pm 0,01^{c}$ & $1,60 \pm 0,02^{b}$ & $1,82 \pm 0,03^{a}$ \\
\hline Lipídios & $3,51 \pm 0,03^{b}$ & $3,65 \pm 0,01^{a}$ & $3,31 \pm 0,04^{c}$ & $3,07 \pm 0,07^{c}$ \\
\hline Fibra Alimentar Total & $1,83 \pm 0,07^{d}$ & $9,61 \pm 0,36^{\mathrm{a}}$ & $6,62 \pm 0,05^{c}$ & $7,59 \pm 0,18^{b}$ \\
\hline Fibra Alimentar Insolúvel & $1,23 \pm 0,02^{d}$ & $6,43 \pm 0,08^{a}$ & $3,34 \pm 0,02^{c}$ & $4,38 \pm 0,03^{b}$ \\
\hline Fibra Alimentar Solúvel & $0,60 \pm 0,05^{b}$ & $3,18 \pm 0,28^{a}$ & $3,28 \pm 0,03^{a}$ & $3,21 \pm 0,15^{a}$ \\
\hline Carboidratos não fibrosos & $71,22 \pm 0,34^{\mathrm{a}}$ & $57,93 \pm 0,43^{c}$ & $61,65 \pm 0,12^{\mathrm{b}}$ & $59,75 \pm 0,50^{d}$ \\
\hline Valor calórico (Kcal/100 g) & $340,46 \pm 0,73^{a}$ & $283,21 \pm 1,63^{c}$ & $294,58 \pm 0,27^{b}$ & $285,95 \pm 1,48^{c}$ \\
\hline
\end{tabular}


Alimentos com elevado valor nutricional e valor calórico reduzido são muito procurados por pessoas que buscam qualidade de vida por meio da alimentação. As formulações enriquecidas com bagaço de maçã, bagaço de laranja e casca de maracujá mostraram-se excelentes alternativas para satisfazer esse perfil de consumidor. A substituição parcial da farinha de trigo pela adição dos subprodutos de frutas contribuiu significativamente para diminuir o aporte calórico e melhorar a qualidade nutricional das formulações, devido ao aumento do teor de fibra. Da mesma forma, constatou-se menor valor energético dessas formulações propostas em comparação a outras de pães de mel enriquecidas com fibra proveniente de cereais (POSSAMAI, 2005). Tais constatações são fundamentadas principalmente pelo fato de que a incorporação das farinhas de frutas reduz a quantidade de carboidratos não fibrosos e calóricos no produto, como o amido, e aumenta consideravelmente o teor de carboidratos complexos pela alta proporção (acima de 50 \%) de fibra alimentar nessas farinhas (Tabela 1). Diferente dos demais carboidratos, a fibra não é degradada pelas enzimas digestivas e assim não serve como fonte energética para o organismo humano. Calcula-se que apenas $7 \%$ da energia derive de açúcares liberados dos polissacarídeos por ação dos micro-organismos intestinais, ou a partir de ácidos graxos de cadeia curta produzidos pela fermentação desses açúcares, os quais são parcialmente absorvidos e usados para a produção de energia (DAMODARAN, PARKIN e FENNEMA, 2010). Vale ressaltar também que a redução expressiva de $18,7 \%$ de carboidratos não fibrosos em relação à FP permitiu menor contribuição calórica da FBM, mesmo apresentando teor superior $(p<0,05)$ de lipídios $(4 \%)$, fato atribuído à presença de quantidade apreciável de gordura $(8,19 \%)$ e fibra $(76,84 \%)$ nesse subproduto de fruta (Tabela 1).

\subsection{ANÁLISE SENSORIAL}

Para garantir sua estabilidade no mercado, o alimento desenvolvido deve apresentar considerável potencial funcional e atender às exigências do consumidor, tanto em relação ao custo do produto, quanto em relação às suas características organolépticas.

A simples adição de teores elevados de fibra em alimentos nem sempre resulta em produtos com características sensoriais agradáveis (DREHER, 1995). Por esse motivo, além da importância de se buscar fontes alternativas de fibra alimentar abundantes e de baixo custo, os testes sensoriais são de fundamental importância para garantir o sucesso do produto desenvolvido.

A Tabela 4 apresenta os valores obtidos na avaliação da aceitabilidade dos pães de mel com incorporação dos diferentes subprodutos de frutas. Nota-se que as três formulações avaliadas pelos 56 julgadores alcançaram boa média de aceitabilidade para todos os atributos sensoriais, com índice de aceitação global (que envolve todos os parâmetros de qualidade sensorial do produto) entre 77,4 \% e $80,3 \%$. Outros estudos sobre a incorporação de farinhas de subprodutos de frutas em formulações alimentícias como biscoitos, bolos e barra de cereais, também resultaram em boa aceitabilidade nos testes sensoriais (PROTZEK, FREITAS e WASCZYNSKJ, 1998; ISHIMOTO et al., 2007; COELHO e WOSIACKI, 2010; ABUD e NARAIN, 2009; SANTOS et al., 2011; ROBERTO, 2011).

Todas as formulações geraram a mesma resposta sensorial independentemente da farinha de fruta incorporada, embora alguns participantes tenham sentido certo sabor residual ou característico das frutas. Vale ressaltar que a adição de farinhas com notáveis diferenças de coloração não interferiu de forma diferenciada na aceitação do produto em relação à esse parâmetro.

Para ser considerado aceito em termos de suas propriedades sensoriais pelo consumidor é necessário que o produto obtenha índice de aceitabilidade de no mínimo $70 \%$ (TEIXEIRA, MEINERT e BARBETTA, 1987). Apesar de estatisticamente insignificante, apenas a nota atribuída ao parâmetro textura para a formulação FBL mostrou-se abaixo desse valor. Talvez o menor aporte de fibra dessa formulação, bem como as características físico-químicas dessa fonte fibrosa, possam ter contribuído para o índice ligeiramente inferior para esse atributo sensorial. 
TABELA 4 - VALORES MÉDIOS DE ACEITABILIDADE REFERENTES AOS ATRIBUTOS AVALIADOS NAS FORMULAÇÕES DE PÃES DE MEL

\begin{tabular}{lccc}
\hline \multirow{2}{*}{ Atributos } & \multicolumn{3}{l}{ Formulações } \\
\cline { 2 - 4 } & FBM & FBL & FCM \\
\hline Cor & $5,80 \pm 0,75^{\mathrm{NS}}$ & $5,85 \pm 0,74^{\mathrm{NS}}$ & $5,57 \pm 0,90^{\mathrm{NS}}$ \\
Aroma & $5,60 \pm 0,77^{\mathrm{NS}}$ & $5,31 \pm 1,25^{\mathrm{NS}}$ & $5,29 \pm 0,92^{\mathrm{NS}}$ \\
Sabor & $5,56 \pm 1,14^{\mathrm{NS}}$ & $5,32 \pm 1,19^{\mathrm{NS}}$ & $5,49 \pm 0,97^{\mathrm{NS}}$ \\
Textura & $5,21 \pm 1,22^{\mathrm{NS}}$ & $4,84 \pm 1,23^{\mathrm{NS}}$ & $5,06 \pm 1,28^{\mathrm{NS}}$ \\
Aceitação Global & $5,62 \pm 1,02^{\mathrm{NS}}$ & $5,42 \pm 0,81^{\mathrm{NS}}$ & $5,45 \pm 0,94^{\mathrm{NS}}$ \\
\hline
\end{tabular}

Médias com letras diferentes na mesma linha diferem entre si $(p \leq 0,05)$ pelo teste de Tukey. NS = médias não diferem significativamente pelo teste de Tukey a $5 \%$ de significância. FBM = formulação adicionada de bagaço de maçã; $\mathrm{FBL}$ = formulação adicionada de bagaço de laranja; FCM = formulação adicionada de casca de maracujá.

Quanto à intenção de compra, as notas atribuídas às formulações testadas (39,3 \% a $44,6 \%$ ) situaram-se na região da escala referente ao termo "possivelmente compraria" (nota 4), indicando intenção favorável de compra dos produtos desenvolvidos (Figura 1).

Considerando a média das notas positivas à compra, 5 e 4, a formulação FBM destacouse com $67,86 \%$ das intenções, seguida pela FBL $(64,29 \%)$ e por último a FCM $(57,14 \%)$. Esse resultado condiz com as melhores médias de aceitabilidade atribuídas à formulação FBM para a maioria dos atributos sensoriais (exceto para o parâmetro cor, embora sem significância estatística).

Sob os pontos de vista nutricional e sensorial, a formulação FBM merece atenção especial. A incorporação de farinha de bagaço de maçã em substituição à farinha de trigo nessa formulação, além de reduzir o valor calórico do produto de forma significativa, possibilitou incremento superior $(p<0,05)$ de fibra alimentar e, ainda, apresentou considerável índice de aceitabilidade $(80,3 \%)$ e maior intenção de compra.

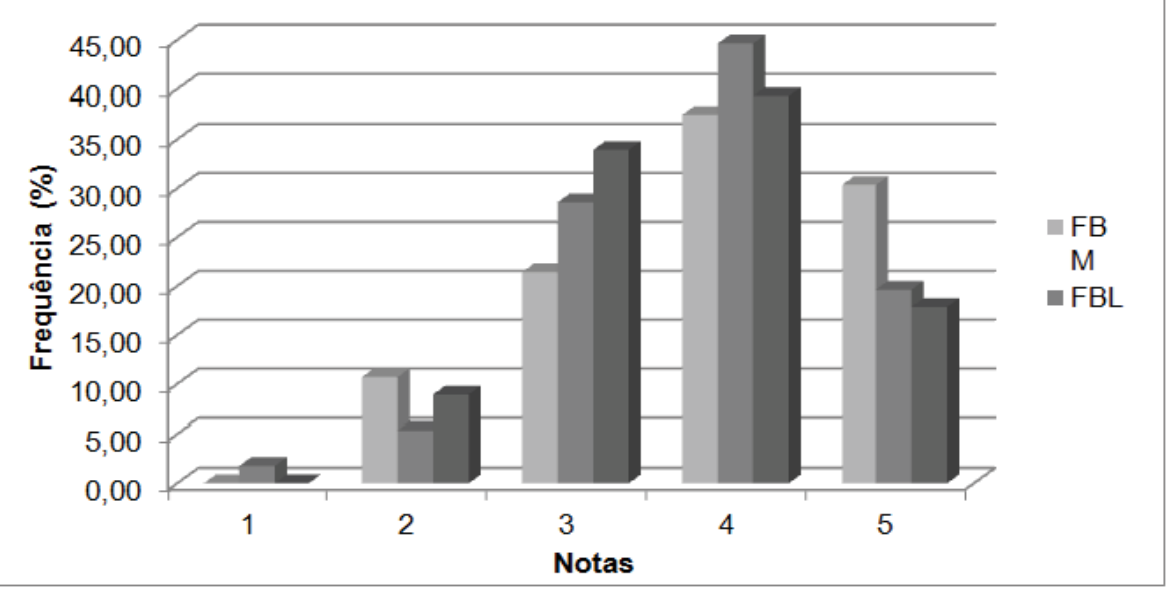

\section{FIGURA 1 - FREQUÊNCIA DAS NOTAS ATRIBUÍDAS ÀS AMOSTRAS DE PÃES DE MEL PARA O TESTE DE INTENÇÃO DE COMPRA}

FBM = formulação adicionada de bagaço de maçã; FBL = formulação adicionada de bagaço de laranja; FCM = formulação adicionada de casca de maracujá. 1 = certamente não compraria, $2=$ possivelmente compraria, $3=$ talvez comprasse/talvez não comprasse, 4 = possivelmente compraria, 5 = certamente compraria.

\section{CONCLUSÃo}

A adição das farinhas dos subprodutos de frutas testadas no presente estudo promoveu 
aumento no teor de fibra alimentar e redução no valor calórico dos pães de mel, que obtiveram boa aceitabilidade e intenção de compra favorável. Dessa forma, viabiliza-se a utilização racional desses subprodutos do processamento de frutas como novas fontes de fibras, evitando o desperdício desses nutrientes potenciais frente à realidade preocupante da carência de alimentação de qualidade para a população de baixa renda. Além disso, os pães de mel enriquecidos com farinhas elaboradas a partir da casca de maracujá, bagaço de maçã e bagaço de laranja possibilitaram a obtenção de produtos funcionais e diferenciados, tornando-se alternativas interessantes para quem busca melhorar o consumo de fibra alimentar, principalmente em relação à sua fração solúvel.

\section{ABSTRACT \\ NUTRITIONAL CHARACTERIZATION AND SENSORY RESPONSE FOR HONEY BREAD WITH HIGH DIETARY FIBER CONTENT PREPARED WITH FLOURS OF BY-PRODUCTS FROM FRUIT PROCESSING}

The objective of this study was to evaluate the nutritional and sensory quality of honey bread enriched with flours made with apple pomace, orange bagasse and passion fruit peel. With the intention of making the use of these alternative and low-cost ingredients feasible as sources of dietary fiber, tests were performed replacing $15 \%$ of wheat flour in the standard formulation by flours of the respective fruit by-products. The test formulations showed high concentrations of dietary fiber, especially the soluble fraction, higher moisture and reduced calorific value. The formulation with added apple pomace stood out from the others due to a significantly higher level of fiber $(9.61 \%)$. The results obtained in the sensory analysis of the honey breads enriched with fiber from fruit by-products indicated good acceptability of these products for all the sensory parameters that were evaluated (color, aroma, flavor and texture), totaling an average index of overall acceptance between $77.4 \%$ and $80.3 \%$. There was also a positive response regarding intent to purchase; $39.3 \%$ to $44.6 \%$ of the responses were within the "would possibly buy" hedonic category with no significant difference between the test formulations. The results indicated the possible use of these fruit by-products as fibrous ingredients in the formulation of various food products, such as honey bread, by significantly improving the fiber content (mainly in relation to the soluble fraction), which would contribute to the prevention of wastage of potential nutrients, as well as improvements in consumer health.

KEY-WORDS: APPLE POMACE; PASSION FRUIT-PEEL; ORANGE BAGASSE; SOLUBLE FIBER; FUNCTIONAL FOOD; SENSORY ANALYSIS.

\section{REFERÊNCIAS}

1 ABUD, A.K.S.; NARAIN, N. Incorporação da farinha de resíduo do processamento de polpa de fruta em biscoitos: uma alternativa de combate ao desperdício. Brazilian Journal of Food Technology, v.12, n.4, p.257-265, 2009

2 AOAC. Association of Official Analytical Chemists. Official methods of analysis of the AOAC international. $16^{\text {th }}$ ed. Washington, 1995. 1018 p.

3 BLIGH, E.C.; DYER, W.J. A rapid method of total lipid. Extraction and purification. Canadian Journal of Biochemistry and Physiology, v. 37, n.52, p. 911-917, 1959.

4 BORTOLUZZI R.C. Aplicação da fibra da polpa da laranja na elaboração de mortadela de frango. 2009. 83 f.Tese (Doutorado em Ciência dos Alimentos), Faculdade de Ciências Farmacêuticas, Universidade de São Paulo, São Paulo, 2009.

5 BRASIL. Ministério da Saúde. Agência Nacional de Vigilância Sanitária. Portaria n. 27 de 13 de janeiro de 1998. Regulamento técnico referente à informação nutricional complementar. Diário Oficial [da] República Federativa do Brasil, Brasília, DF, 16 de janeiro de 1998, Seção I.

6 BRASIL. Ministério da Saúde. Agência de Vigilância Sanitária. RDC n. 360 de 23 de dezembro de 2003. Regulamento técnico sobre rotulagem nutricional de alimentos embalados, tornando obrigatória a rotulagem nutricional. Diário Oficial [da] República Federativa do Brasil, Brasília, DF, 26 de dezembro de 2003, Seção I, p. 33-44.

7 CANTERI, M.H.G. Caracterização comparativa entre pectinas extraídas do pericarpo de maracujá-amarelo (Passiflora edulis $\mathrm{f}$. flavicarpa). 2010. $162 \mathrm{f}$. Tese (Doutorado em Tecnologia de Alimentos), Setor de Tecnologia de Alimentos, Universidade Federal do Paraná, Curitiba, 2010.

8 CANTERI, M.H.G.; MORENO, L.; WOSIACKI, G.; SCHEER, A. Pectina: da matéria-prima ao produto final. Polímeros, v.22, n.2, p. 149-157, 2012.

9 CERQUEIRA, P.M.; FREITAS, M.C.J.; PUMAR, M.; SANTANGELO, S.B. Efeito da farinha de semente de abóbora (Curcubita maxima, L.) sobre o metabolismo glicídico e lipídico em ratos. Revista de Nutrição, v.21, n.2, p.129-136, 2008.

10 COELHO, L.M.; WOSIACKI, G. Avaliação sensorial de produtos panificados com adição de farinha de bagaço de maçã. Ciência e Tecnologia de Alimentos, v.30, n.3, p. 582-588, 2010. 
11 DAMODARAN, S.; PARKIN, K.L.; FENNEMA, O.R. Química de alimentos de Fennema. 4.ed. Porto Alegre: Artmed, 2010. $900 \mathrm{p}$

12 DREHER, M.L. Food industry perspective: functional properties and food uses of dietary fiber. In: KRITCHEVSKY, D.; BONFIELD, C. (Eds). Dietary fiber in heath \& disease. Minnesota: Eagan Press, 1995. p. 467-474.

13 ELLEUCH, M.; BEDIGIAN. D.; ROISEUX, O.; BESBES, S. BLECKER, C.; ATTIA, H. Dietary fibre and fibre-rich byproducts of food processing- characterisation, technological functionality and commercial applications: a review. Food Chemistry, v.124, p.411-421, 2011.

14 FIETZ V. R.; SALGADO J.M. Efeito da pectina e da celulose nos níveis séricos de colesterol e triglicerídeos em ratos hiperlipidêmicos. Ciência e Tecnologia de Alimentos, v.19, n.3, p. 318-321, 2010.

15 GIUNTINI, E.B.; LAJOLO, F.M.; MENEZES, E.W. Potencial de fibra alimentar em países iberoamericanos: alimentos, produtos e resíduos. Archivos Latinoamericanos de Nutrición, v.53, n.1, p.14-20, 2003.

16 GONÇALVES, M.C.R.; COSTA, M.J.C.; ASCIUTTI, L.S.R.; DINIZ, M.F.F.M. Fibras dietéticas solúveis e suas funções nas dislipidemias. Revista Brasileira de Nutrição Clínica, v.22, n.2, p.167-173, 2007.

17 Instituito Adolfo Lutz. Análise sensorial. In: MÉTODOS físicoquímicos para análise de alimentos. São Paulo, 2008. p. 279-320.

18 ISHIMOTO, F.Y; HARADA, A.I.; BRANCO, I.G.; CONCEIÇÃO, W.A.S.; COUTINHO, M.R. Aproveitamento alternativo da casca do maracujá- amarelo (Passiflora edulis f. var. flavicarpa Deg.) para produção de biscoitos. Revista Ciências Exatas e Naturais, v.9, n.2, p. 279-292, 2007.

19 LARRAURI, J.A. New approaches in the preparation of high dietary fibre powders from fruit by-products. Trends in Food Science \& Technology, v.10, n.1, p. 3-8, 1999.

20 LÓPEZ, G.; ROS, G.; RINCÓN, F.; PERIAGO, M.J.; MARTíNEZ, C.; ORTUÑO, J. Propiedades funcionales de la fibra dietética: mecanismos de acción en el tracto gastrointestinal. Archivos Latino Americanos de Nutrition, v.47, n.3, p. 203-207, 1997.

21 MATTOS, L.L.; MARTINS, I.S. Consumo de fibras alimentares em população adulta. Revista Saúde Pública, v.34, n.1, p. $50-55,2000$.

22 MAURO, A.K.; SILVA, V.L.M.; FREITAS, M.C.J. Caracterização física, química e sensorial de cookies confeccionados com farinha de talo de couve (FTC) e farinha de talo de espinafre (FTE) ricas em fibra alimentar. Ciência e Tecnologia de Alimentos, v.30, n.3, p. 719-728, 2010.

23 MENEZES, E.W.; GIUNTINI, E.B.; LAJOLO, F.M. Perfil da ingestão de fibra alimentar e amido resistente pela população brasileira nas últimas três décadas. In: LAJOLO, F.M.; SAURA-CALIXTO, F.; PENNA, E.W.; MENEZES, E.W. Fibra dietética em Iberoamérica - tecnología y salud: obtención, caracterización, efecto fisiológico y aplicación en alimentos. São Paulo: Livraria Varela, 2001. p. 433-444.

24 Núcleo de Estudos e Pesquisa em Alimentação (NEPA). Universidade Estadual de Campinas. Tabela brasileira de composição de alimentos (TACO). 4.ed. Campinas: Book Editora, 2011. $164 \mathrm{p}$.

25 PEREZ, P.M.P.; GERMANI, R. Elaboração de biscoitos tipo salgado, com alto teor de fibra alimentar, utilizando farinha de berinjela (Solanum melongena, L.). Ciência e Tecnologia de Alimentos, v.27, n.1, p.186-192, 2007.

26 POSSAMAI, T.N. Elaboração do pão de mel com fibra alimentar proveniente de diferentes grãos, sua caracterização físico-química, microbiológica e sensorial. 2005.69 f. Dissertação (Mestrado em Tecnologia de Alimentos), Setor de Tecnologia, Universidade Federal do Paraná, Curitiba, 2005.

27 PROTZEK, E.C; FREITAS, R.J.S; WASCZYNSKJ, N. Aproveitamento do bagaço de maçã na elaboração de biscoitos ricos em fibra alimentar. Boletim do CEPPA, v.16, n.2, p.263-275, 1998.

28 RAUPP, D.S.R.; CARRIJO, K.C.R.; COSTA, L.L.F.; MENDES, S.D.C.; BANZATTO, D.A. Propriedades funcionaisdigestivas e nutricionais de polpa-refinada de maçã. Scientia Agricola, v.57, n.3, p. 395-402, 2000.

29 RIQUE, A.B.R.; SOARES, E.A.; MEIRELLES, C.M. Nutrição e exercício na prevenção e controle das doenças cardiovasculares. Revista Brasileira de Medicina do Esporte, v.8, n.6, p.244- 254, 2002.

30 ROBERTO, B. S. Resíduo de goiaba: metabolismo em ratos e aplicabilidade em barras de cereais. 2012.163 f. Dissertação (Mestrado em Ciência e Tecnologia dos Alimentos), Universidade Federal de Santa Maria, Santa Maria, 2012 .

31 SANTOS, A.A.O.; SILVA, I.V.C.; SANTOS, J.P.A.; SANTANA, D.G.; ALMEIDA, M.L.; MARCELLINI, P.S. Elaboração de biscoitos de chocolate com substituição parcial da farinha de trigo por polvilho azedo e farinha de albedo de laranja. Ciência Rural, v.41, n.3, p.531-536, 2011.

32 SILVA, M.R.; BORGES, S.; MARTINS, K.A. Avaliação química, física e sensorial de biscoitos enriquecidos com farinha de jatobá-do-cerrado e de jatobá-da-mata como fonte de fibra alimentar. Brazilian Journal of Food Technology, v.4, n.73, p.163-70, 2001.

33 SPSS Incorporation. Statistical package for the social sciences for windows (SPSS). Version 8.0. Chicago, 1997. TEIXEIRA, E.; MEINERT, E.M.; BARBETTA, P.A. Análise sensorial de alimentos. Florianópolis: UFSC, 1987.182 p. 